\title{
DISTRIBUCIÓN DE ROGADINAE (HYMENOPTERA: BRACONIDAE) EN UNA GRADIENTE ALTITUDINAL EN LOS ANDES DEL SUR DEL PERÚ
}

\section{DISTRIBUTION OF ROGADINAE (HYMENOPTERA: BRACONIDAE) IN AN ALTITUDINAL GRADIENT IN THE SOUTHERN PERUVIAN ANDES}

\author{
Lidia A. Sulca Garro ${ }^{1}$
}

\begin{abstract}
Resumen
Se estudió la variación altitudinal de la riqueza, diversidad y estructura comunitaria de las avispas parasitoides de la subfamilia Rogadinae (Hymenoptera: Braconidae) en una gradiente altitudinal en el Valle de Cosñipata (Cuzco, Perú). Para ello, se definieron cuatro sitios de estudio entre 800 y 2800 m: Tono (800 m), San Pedro (1500 m), Rocotal (2000 m) y Wayquecha (2800 m) en los que se efectuaron cinco muestreos empleando trampas Malaise. Se capturó un total de 351 individuos comprendiendo 109 morfoespecies en 5 géneros (Aleiodes, Cystomastax, Clinocentrus, Triraphis y Stiropius).

La localidad San Pedro (1500 m) presentó mayor índice de diversidad de Shannon-Wiener (H), seguida por Tono, Rocotal y Wayquecha, con valores muy cercanos de diversidad (H: 3.894, 3.062, 2.989, 2.653, respectivamente). El índice de similaridad de Bray-Curtis muestra mayor similitud entre San Pedro y Rocotal (0.29). La composición de especies estuvo correlacionada positivamente con la elevación.

Palabras clave: Diversidad, bosque nublado, avispas parasitoides, valle de Cosñipata.
\end{abstract}

\begin{abstract}
We studied the altitudinal variation in richness, diversity and community structure of parasitoid wasps of the Rogadinae subfamily (Hymenoptera: Braconidae), along an elevational gradient in the Cosñipata valley (Cuzco, Peru).

We selected four open field collecting localities at several elevations from 800 to $2800 \mathrm{~m}$ : Tono (800 m), San Pedro (1500 m), Rocotal (2000 m) and Wayquecha (2800 m). We carried out five collections using Malaise traps. We captured a total of 351 individuals: 109 morphospecies in 5 genera (Aleiodes, Cystomastax, Clinocentrus, Triraphis and Stiropius).

San Pedro $(1500 \mathrm{~m})$ presented the highest diversity level, according to the Shannon-Wiener index $(\mathrm{H})$, followed by Tono, Rocotal and Wayquecha $(\mathrm{H}: 3.894,3.062,2.989,2.653$ respectively). The Bray Curtis similarity index showed the greatest similarity between San Pedro and Rocotal (0.29). Species composition was positively correlated with elevation.
\end{abstract}

Key words: Diversity, cloud forest, parasitoid wasps, Cosñipata valley.

\section{Introducción.}

La relación existente entre la diversidad y la elevación es un patrón ecológico frecuentemente documentado pero polémico, debido a que no han podido desarrollarse generalizaciones o explicaciones universales del fenómeno (Rahbek, 1995). Esto se debe a que el comportamiento de la curva de riqueza de especies es muy variado, ya que puede diferir entre taxones idénticos o disímiles y cambiar local o regionalmente (Rahbek, 1995). Huston (1994) también menciona a la altitud como un factor importante en la riqueza y composición de especies.

Existen dos patrones generales de la asociación entre altitud y riqueza de especies: una disminución de la riqueza con el aumento de la elevación (Rahbek, 1973,1976; McCoy, 1990; Lobo \& Halffter, 2000) o una relación en forma de “joroba”, donde los valores máximos de riqueza se observan en altitudes medias (Janzen, 1973, 1976; Olson, 1994; Fagua, 1999; Escobar et al., 2005).

El presente, es el primer estudio de distribución altitudinal y diversidad de avispas parasitoides bracónidos en el Perú. Gomes (2005) estudió la diversidad de Braconidae en una gradiente altitudinal en Campos do Jordão (Brasil) encontrando el pico de abundancia y riqueza en las altitudes intermedias. Terborgh (1971) realizó un estudio de distribución en gradiente altitudinal de la avifauna en la cordillera de Vilcabamba (Perú) donde plantea que las causas que limita la distribución de las especies son principalmente los factores físicos, competencia y presencia de ecotonos. 
En el Valle de Cosñipata, la vegetación es del tipo bosque montano tropical. Este tipo de bosque representa un raro y frágil ecosistema que está en peligro en muchas partes del mundo, donde frecuentemente se encuentran especies endémicas y amenazadas, ha demostrado un rol fundamental en la preservación de agua (Bubb et al., 2004). Las especies de selva tropical con distribución limitada a estrechos cinturones altitudinales pueden ser particularmente sensibles a la pérdida de hábitat (p.ej. deforestación) y a la fragmentación, lo cual puede limitar significativamente el desplazamiento de las especies (Olson, 1994).

La familia Braconidae es la segunda más grande del orden Hymenoptera, con 36 subfamilias, 1000 géneros y más de 17000 especies descritas para el mundo (Yu et al., 2005). Son agentes reguladores de diversos grupos de insectos fitófagos, sirviendo como indicadores de la presencia o ausencia de estas poblaciones (La Salle \& Gauld, 1993). Además, dado su enorme espectro de estrategias, conforman uno de los principales grupos de interés en la investigación de la evolución de las interacciones parasitoidehospedero, así como de la simbiosis con virus (Campos \& Sharkey, 2006).

El objetivo principal del presente estudio es proporcionar información sobre la distribución de la subfamilia Rogadinae (Braconidae) en el valle de Cosñipata, para lo cual se estudió la variación altitudinal de la riqueza, diversidad y estructura comunitaria de dichas avispas parasitoides. Al ser el primer estudio en gradiente altitudinal de Braconidae para el Perú, es difícil especular sobre las posibles tendencias que existan entre la diversidad de Rogadinae con la altitud. Basándonos en estudios de Gomes (2005), se hipotetiza que existe mayor diversidad en altitud intermedias entre las especies de Rogadinae.

\section{Materiales y métodos.}

Área de estudio.

Este estudio fue realizado en el Valle de Cosñipata, provincia de Paucartambo, departamento de Cuzco.

El valle abarca la zona límite entre el Parque Nacional del Manu y su zona de amortiguamiento. Los puntos de muestreo se ubicaron entre los 800 y $2800 \mathrm{~m}$ de altitud. Según la clasificación de Holdridge estos pertenecen a: bosque muy húmedo pre-montano subtropical (bmh-PM) en Tono (12 $57^{\circ}$ - 71 $32^{\circ} \mathrm{W}$, $800 \mathrm{~m})$, y bosque montano muy húmedo subtropical

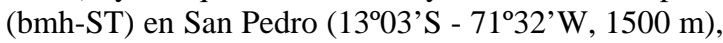

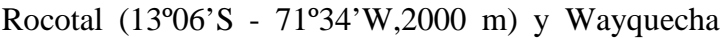

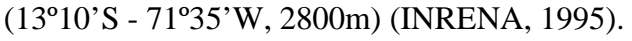

Método de colecta.

Las colectas se realizaron de junio 2007 a enero 2008, empleando 12 trampas Malaise. Se colocaron tres trampas en cada lugar: Tono (800 m), San Pedro
(1500 m), Rocotal (2000 m) y Wayquecha (2800 m). Se realizó el recambio del contenido del frasco colector en periodos entre 30 a 40 días con un total de 5 muestreos por cada localidad, a excepción de Rocotal debido a problemas climatológicos, no se pudo realizar un muestreo óptimo ya que las trampas no funcionaron el primer mes afectando de alguna manera los resultados.

Dichos contenidos fueron depositados en bolsas de polipropileno con alcohol $96^{\circ}$ y trasladadas al Departamento de Entomología del Museo de Historia Natural de la Universidad Nacional Mayor de San Marcos (MUSM), donde se procedió a separar las avispas para su montaje, etiquetado e identificación con la ayuda de claves y posteriormente depositadas. Análisis Estadísticos.

Para la cuantificación de diversidad de Rogadinae en nuestro estudio, se utilizaron los índices de diversidad Shannon-Wiener y Simpson para cada sitio. Estos fueron calculados con el software PAST (Hammer et al., 2001). Se comparó la similaridad de la composición de especies en cada sitio mediante el índice de similitud de Bray Curtis. Así mismo, para analizar la correlación entre la altitud y la composición de especies se calculó el Test de Mantel con el programa $\mathrm{R}$ versión 2.5.1.

Por último, se elaboró una curva de rarefacción con el programa Past (Hammer et al., 2001), ya que se emplea para hacer la comparación de números de especies entre comunidades cuando los tamaños de las muestras no son iguales (Moreno, 2001) como fue nuestro caso.

\section{Resultados.}

Se capturaron 351 individuos, comprendiendo 109 morfoespecies en 5 géneros (Aleiodes, Cystomastax, Clinocentrus, Triraphis y Stiropius).

Aleiodes fue el género con mayor número de morfoespecies (61), presente en todos los lugares y predominantemente en San Pedro (1500 m) con 29 morfoespecies (Figura 1). El número de morfoespecies Triraphis fue mayor en San Pedro y menor en Wayquecha $(2000 \mathrm{~m})$ donde presentó solo una morfoespecie. Clinocentrus estuvo presente a partir de los $1500 \mathrm{~m}$ (San Pedro), donde presentó el mayor número de morfoespecies. Stiropius se encontró presente en todas las altitudes pero en bajo número. Finalmente, se determinó solamente una Cystomastax en Tono y San Pedro (Figura 1). En el 5to muestreo fue donde se presentó mayor número de morfoespecies.

Finalmente, el test de Mantel muestra que existe una correlación $(0,044)$ entre la altitud y composición de especies $(r=0,7033, \mathrm{P}<0,05)$.

San Pedro $(1500 \mathrm{~m})$ presentó mayores índices de diversidad Shannon-Wiener y Simpson con 3.894 y 0.976 respectivamente a diferencia de Wayquecha que 
presentó los menores índices 2.653 y 0.901 respectivamente (Tabla 1 ).

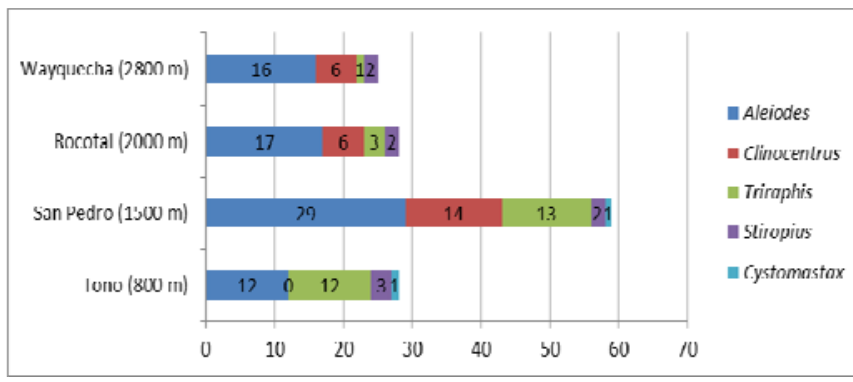

Figura 1. Número de morfoespecies de los géneros de Rogadinae respecto del nivel altitudinal.

Tabla 1. Índices de Diversidad de morfoespecies de Rogadinae.

\begin{tabular}{lcccc}
\hline & $\begin{array}{c}\text { Tono } \\
(800 \mathrm{~m})\end{array}$ & $\begin{array}{c}\text { San Pedro } \\
(1500 \mathrm{~m})\end{array}$ & $\begin{array}{c}\text { Rocotal } \\
(2000 \mathrm{~m})\end{array}$ & $\begin{array}{c}\text { Wayquecha } \\
(2800 \mathrm{~m})\end{array}$ \\
\hline Taxa_S & 28 & 59 & 28 & 25 \\
Individuals & 48 & 126 & 60 & 117 \\
Shannon_H & 3.062 & 3.894 & 2.989 & 2.653 \\
Simpson_1-D & 0.9332 & 0.9761 & 0.9222 & 0.9012 \\
\hline
\end{tabular}

La curva de rarefacción muestra que San Pedro (1500 m) presenta mayor riqueza específica esperada y abundancia que Tono, Rocotal y Wayquecha (Figura 2).

San Pedro (1500 m) y Rocotal (2000 m) presentan el índice de similaridad con 0.29 de similitud. Los lugares con índice de similaridad más bajo son Tono $(800 \mathrm{~m})$ y Wayquecha $(2800 \mathrm{~m})$ con 0.012 (Tabla 2) (Figura 3).

Tabla 2. Índice de similaridad de Bray-Curtis.

\begin{tabular}{lcccc}
\hline & $\begin{array}{c}\text { Tono } \\
(800 \mathrm{~m})\end{array}$ & $\begin{array}{c}\text { San Pedro } \\
(1500 \mathrm{~m})\end{array}$ & $\begin{array}{c}\text { Rocotal } \\
(2000 \mathrm{~m})\end{array}$ & $\begin{array}{c}\text { Wayqecha } \\
(2800 \mathrm{~m})\end{array}$ \\
\hline Tono $(800 \mathrm{~m})$ & & & & \\
San Pedro $(1500 \mathrm{~m})$ & 0.12644 & & & \\
Rocotal $(2000 \mathrm{~m})$ & 0.037037 & 0.29032 & & \\
Wayqecha $(2800 \mathrm{~m})$ & 0.012121 & 0.074074 & 0.13559 & \\
\hline
\end{tabular}

Los resultados muestran que existen muy pocas especies abundantes 3.67\%, muchas especies intermedias $37.61 \%$ (entre 3-14 individuos), muchas especies únicas $44.5 \%$ y con dos individuos $17.43 \%$ (Tabla 3).

\section{Discusión.}

Aleiodes fue el género que predominó en todos los lugares estudiados, con un total de 160 especímenes y 61 morfoespecies. Esto concuerda con lo señalado por Shaw et al. (1997, 1998), quienes señalan a éste género como el de mayor diversidad dentro de los rogadinos. Aleiodes y Triraphis son los géneros más comunes en el Neotrópico, sin embargo muchas especies aún no han sido descritas y se desconoce de especies.

detalles de su biología. Se estima que las especies no descritas de Aleiodes en el Neotrópico superan las 200 especies (Delfín-González \& Wharton, 2002; Townsend \& Shaw, 2009). Cystomastax fue el género menos representado en este estudio, según la literatura es poco común en el Neotrópico (Shaw, 1997). Clinocentrus estuvo ausente en Tono (800 m). Esto nos indicaría que su hospedero no está presente o tiene una población muy baja en ese lugar ya que las avispas parasitoides están relacionadas a la presencia y/o ausencia de sus hospederos. Stiropius se presentó en todas las altitudes pero en bajo número. Esto podría deberse a que las trampas malaise no serían eficientes en su captura. En época de lluvias se colectó mayor cantidad de individuos, posiblemente se deba al aumento de la población de estos parasitoides. Los géneros Clinocentrus y Stiropius son nuevos registros para Perú.

Se comprobó que existe una correlación entre la altitud y la composición de especies de los rogadinos en Cosñipata. Al comparar la similitud de especie en los diferentes niveles altitudinales estudiados fue muy baja, es decir, Tono, San Pedro, Rocotal y Wayquecha no tienen la misma composición

Así mismo, la similitud de especies en los diferentes niveles altitudinales estudiados fue muy baja, es decir que cada lugar tiene una composición de especies particular. Esto podría deberse a que la altitud actúa como barrera geográfica en la distribución de las especies de rogadinos y sus hospederos. Los factores climáticos son las variables relacionadas con la altitud que estén actuando como limitantes de la distribución de especies. Huston (1994) señala que la altitud es una variable que cambia la frecuencia en la riqueza y composición de especies de igual modo además, la altitud se relaciona a cambios en la composición de las especies de artrópodos (Janzen, 1973).

El valle de Cosñipata presenta alta diversidad de especies. Esto se debería a que este valle es un bosque nublado y tiene como característica concentrar una alta diversidad en diferentes grupos de organismos (Bubb et al., 2004),

Tabla 3. Porcentaje de individuos colectados.

\begin{tabular}{lc}
\hline No de individuos & Porcentaje \\
\hline$>15$ individuos & 3.67 \\
10 - 14 individuos & 2.75 \\
06 - 09 individuos & 6.42 \\
03 - 05 individuos & 24.77 \\
02 individuos & 17.43 \\
01 individuos & 44.5 \\
Total = 109 individuos & $100 \%$ \\
\hline
\end{tabular}


además de presentar escasa disturbación antropogénica. San Pedro presentó el mayor índice de diversidad.

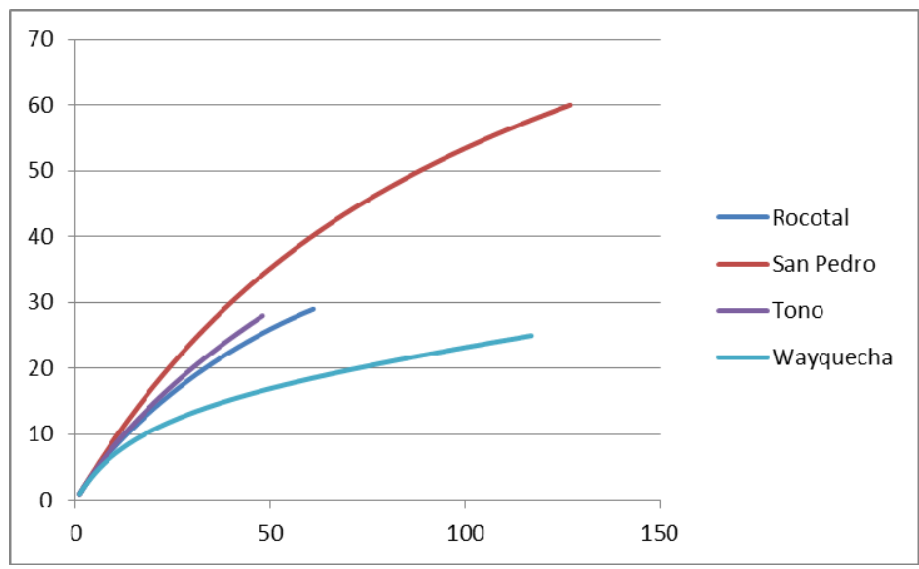

Figura 2. Curvas de Rarefacción para los diferentes niveles altitudinales: Rocotal (2000 m), San Pedro (1500 m), Tono (800 m) y Wayquecha (2800 m).

La curva de rarefacción señala que San Pedro $(1500 \mathrm{~m})$ presenta mayor riqueza específica, otorgándole al patrón de distribución altitudinal de la subfamilia Rogadinae una forma de “joroba” ya que los valores máximos de riqueza de especies se observan en altitudes intermedias.

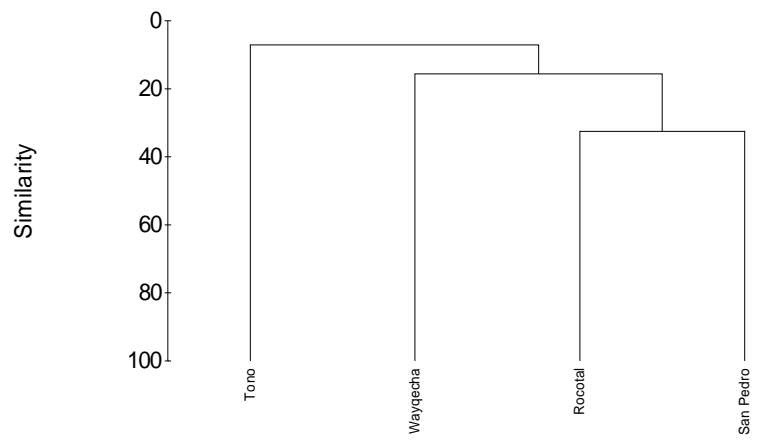

Figura 3. Dendrograma de Bray- Curtis para los diferentes niveles altitudinales: Rocotal (2000 m), San Pedro (1500 m), Tono (800 m) y Wayquecha (2800 m).

Este patrón es explicado por Rahbek (1997), el encontró que la mayor riqueza de especies en altitudes intermedias se debe a la superposición de los rangos de distribución de las especies en las altitudes intermedias ya que la extensión de sus rangos altitudinales está limitada por la elevación máxima y mínima posible en la región. Sin embargo, la distribución a lo largo de una gradiente altitudinal de los insectos puede variar según los taxones ya que presentan una variedad de estrategias adaptativas frente a los cambios ambientales (Colwell \& Lees, 2000; Colwell et al., 2005).

Rahbek (1995), señala que existe polémica en los resultados de investigaciones sobre la distribución altitudinal de los insectos en una gradiente altitudinal y sigue siendo insuficiente sugerir un modelo uniforme de riqueza de especies en relación con la elevación, por lo que no se puede generalizar, esto depende del grupo de insectos y del lugar donde se estudia.

Con respecto a la abundancia proporcional de especies, los resultados muestran un elevado número de “singletons" y “doubletons", se puede deber a que Rogadinae es muy diverso con poca abundancia de individuos por lo que es necesario un prolongado tiempo de muestreo. Resultados similares se han presentado en la familia Ichneumonidae (considerado grupo hermano de Braconidae) con un elevado número de especies y muy pocos individuos, obtenidos por Bartlett et al. (1999) en Costa Rica, Belize y Panamá; y se debe a que existe mucha diversidad taxonómica, pero poca abundancia de individuos (Rodríguez, 2006). Así mismo, Magurran (1988) señala a este tipo de distribución como una distribución logarítmica y es concordante con insectos que habitan en áreas naturales más o menos equilibradas, o con disturbio moderado.

\section{Agradecimientos.}

La Asociación para la Conservación de la Cuenca Amazónica por financiar el trabajo de campo. A la Fundación Ideawild por financiar los materiales de campo y de laboratorio. Al Ministerio del Ambiente que brindó el permiso de colecta. A Carol Castillo, Carlos Peña y Gerardo Lamas quienes nos brindaron sus comentarios y sugerencias.

\section{Literatura citada.}

Bartlett R., Pickeringgauld J., Gauld I. \& Windsor D. 1999. Estimating global biodiversity, tropical beetles and wasps send different signals. Ecological Entomology. 24: 118 - 121.

Bubb P., May I., Miles L. \& Sayer J. 2004. Cloud forest agenda. UNEP-WCMC Biodiversity Series No 20. Cambridge, UK. 36pp.

Campos D.F. \& Sharkey M.J. 2006. Familia Braconidae .En: Fernández F. \& Sharkey M. (eds.). Introducción a los Hymenoptera de la Región Neotropical .Bogotá D.C.: Sociedad Colombiana de Entomología y Universidad Nacional de Colombia, 331-384. 
Castillo C., Sääksjärvi I., Bennett A.M.R. \& Broad G.R. 2012. First record of Acaenitinae (Hymenoptera, Ichneumonidae) from South America with description of a new species and a key to the world species of Arotes Gravenhorst. ZooKeys 137: 77-88.

Colwell R.K. \& Lees D.C. 2000. The mid-domain effect: geometric constraints on the geography of species richness. Trends in Ecology and Evolution 15:70-76.

Colwell R.K., Rahbek C. \& Gotelli N. 2005. The middomain effect: there's a baby in the bathwater. American Naturalist.166:149-154.

Delfín-González H. \& Wharton. R.A. 2002. Distribution of species and species-groups of Aleiodes (Hymenoptera: Braconidae) in Mexico. Folia Entomología Mexicana. 41(2):215-227.

Escobar E., Lobo J.M. \& Halffter G. 2005. Altitudinal variation of dung beetle (Scarabaeidae: Scarabaeinae) assemblages in the Colombian Andes. Global Ecology and Biogeography.14:327-337.

Fagua G. 1999. Capitulo XI. Variación de las mariposas y hormigas de un gradiente altitudinal de la Cordillera Oriental (Colombia), En: Amat, G.; Andrade-C. M. G. Y Fernández, E (eds.). Insectos de Colombia Vol. 2. Bogotá D.C: Academia Colombiana de Ciencias Exactas, Físicas y Naturales Colección Jorge Álvarez Lleras. 13: 317-362.

Gomes A. 2005. A fanuna Braconidae (Ichneumonidae) na região de Campos do Jordão, Sao Paulo, Brasil. Tesis Doctoral. Universidad Fereral de São Carlos.

Hammer O., Harper D.A.T \& Ryan P.D.2001.Past: Paleontological Statistics software package for education and data. Palaeontologia Electronica 4(1): 9.

Huston M.A.1994. Biological diversity. The coexistence of species on changing landscapes. Cambridge University Press, Cambridge, UK. 681 pp.

Janzen D.H. 1973. Sweep samples of tropical foliage insects: effects of seasons, vegetation types, elevation, time of day, and insularity. Ecology.54: 687-708.

Janzen D.H, Ataroff M. , Farinas, M ,et al.1976. Changes in the arthropod community along an elevational transect in the venezuelan Andes. Biotropica .3:193-203.

Janzen D.H. 1981. The Peak in North American Ichneumonid species richness lies between 380 and 420 N. Ecology. 62 (3): 532 - 537.

Lobo J.M. \& G. Halffter. 2000. Biogeographical and ecological factors affecting the altitudinal variation of mountainous communities of coprophagous beetles (Coleoptera, Scarabeoidea): a comparative study .Annals of Entomological Society of America.39: 115-126.

Magurran A.E. 1988. Ecological diversity and its measurement. Princeton University Press, New Jersey, 179 pp.

McCoy E. 1990. The distribution of insects along elevational gradients. Oikos. 58: 313-322.

Moreno C. 2001. Métodos para medir la biodiversidad. MyT-Manuales y Tesis. Orcyt - Unesco y Sociedad Entomológica Aragonese. 86p.
Instituto Nacional de Recursos Naturales (INRENA). 1995. Mapa Ecológico del Perú.219pp.

La Salle J. \& Gauld I. 1993. Hymenotera and biodiversity. CAB International. 348pp.

Olson D. 1994. The Distribution of Leaf Litter Invertebrates Along a Neotropical Altitudinal Gradient. Journal of Tropical Ecology.10 (2): 129-150.

Rahbek C. 1995. The elevational gradient of species richness: a uniform pattern?. Ecography .18: 200-205.

Rahbek C. 1997. The relationship among Area, Elevation , and Regional Species Richness in Neotropical Birds. The American Naturalist. 149:875 -902.

Rodriguez A. 2006. Estudio de la Fauna de Ichneumonidae Cenobionte (Hymenoptera) como potenciales bioindicadores de diversidad en ecosistemas de montaña mediterráneas. Tesis Doctoral. Universidad de Alicante, Alicante.

Sanders N.J. 2002. Elevational gradients in ant species richness: area, geometry, and Rapoport's rule. Ecography. 25 : 25-32.

Sääksjärvi I.E. 2003.Diversity of parasitic wasps (Hymenoptera: Ichneumonidae) in a lowland rain forest mosaic in Peruvian Amazonia. Annales Universitatis Turkuensis Series A II 169.PhD thesis, University of Turku, Turku.

Shaw S. 1997. Subfamilia Rogadinae. En: Wharton R.A., P.M. Marsh \& M.J. Sharkey (eds.). Manual para los géneros de la famlia Braconidae (Hymenoptera) del Nuevo Mundo. The International Society of Hymenopterists, Washington D.C.411-420.

Shaw S.R., Marsh P.M. \& Fortier J.C. 1997. Revision of North American Aleiodes (part 1): the pulchripes Wesmael species-group in the New World (Hymenoptera: Braconidae: Rogadinae). Journal of Hymenoptera Research. 6: 10-35.

Shaw S.R., Marsh P.M. \& Fortier J.C. 1998. Revision of North American Aleiodes (part 2): the apicalis Brulle species-group in the New World (Hymenoptera: Braconidae: Rogadinae). Journal of Hymenoptera Research.7 (1):62-73.

Terborgh J.1971. Distribution on environmental gradientes: Theory and a preliminary interpretation of distributional patterns in the avifauna of the Cordillera Vilcabamba,Peru. Ecology 52: 23-40.

Townsend A.C \& Shaw S.R. 2009. Nine new species of Aleiodes Wesmael reared at Yanayacu Biological Station (Hymenoptera: Braconidae:Rogadinae) in eastern Ecuador. Journal of Insect Science 9(33): 1-21.

Wharton R.A., Marsh P.M. \& Sharkey M.J. 1997. Manual para los géneros de la familia Braconidae (Hymenoptera) del Nuevo Mundo. The International Society of Hymenopterists, Washington D.C.

Yu D., Van Achterberg C. \& Horstann K. 2005. World Ichneumonoidea 2004: taxonomy, biology, morphology and distribution. Electronic Compact Disk. Taxapad, Vancouver, Canada.

\footnotetext{
${ }^{1}$ Departamento de Entomología, Museo de Historia Natural. Universidad Nacional Mayor de San Marcos. Av. Arenales 1256. Apartado 14-0434, Lima-14, Perú. Centro de Ecología y Biodiversidad, Loma Bella 240. Surco. Lima-33, Perú. E-mail: lidia.sulca@gmail.com
} 